\title{
Insulin sensitivity during acute elevation of NEFA: influence of fat composition and gender
}

\author{
K. J. Newens, A. K. Thompson, A. Przemska, D. Vasilopoulou, A. L. Turner, K. G. Jackson \\ and C. M. Williams \\ Hugh Sinclair Unit of Human Nutrition, University of Reading, RG6 6AP, UK
}

Conditions associated with impairments in insulin sensitivity (SI), such as obesity and type-II diabetes (T2D), often present with elevated NEFA. While lipid infusion studies have shown that increasing NEFA can impair glucose metabolism ${ }^{(1)}$, there are relatively few studies investigating the effect of NEFA elevation following fat loads of differing composition.

Sixty healthy volunteers participated in a single-blind crossover trial. Subjects were randomly assigned to either $0.75 \mathrm{~g} / \mathrm{kg}$ bodyweight (bw) of palm stearin (SFA) or $0.65 \mathrm{~g} / \mathrm{kg}$ bw of palm stearin and $0.1 \mathrm{~g} / \mathrm{kg}$ bw of DHA-rich fish oil ( $n-3$ PUFA) on separate occasions; study visits for females were conducted at 4-week intervals to control for the menstrual cycle. The oils were emulsified into chocolate drinks and given as a bolus at baseline $(0 \mathrm{~min})$, followed by smaller drinks every $30 \mathrm{~min}$. At $60 \mathrm{~min}$, an infusion of heparin $(500 \mathrm{IU}$ bolus $+0.4 \mathrm{IU} /$ $\mathrm{kg} \mathrm{bw} / \mathrm{min}$ ) was administered to activate lipoprotein lipase ${ }^{(2)}$. At $240 \mathrm{~min}$, a hyperinsulinaemic (HI)-euglycaemic clamp (1 mU/kg/min) was initiated. SI was calculated as the mean glucose infusion rate for the last $30 \mathrm{~min}$ of the $150 \mathrm{~min}$ clamp, either adjusted for bw or fat-free mass (ffm).

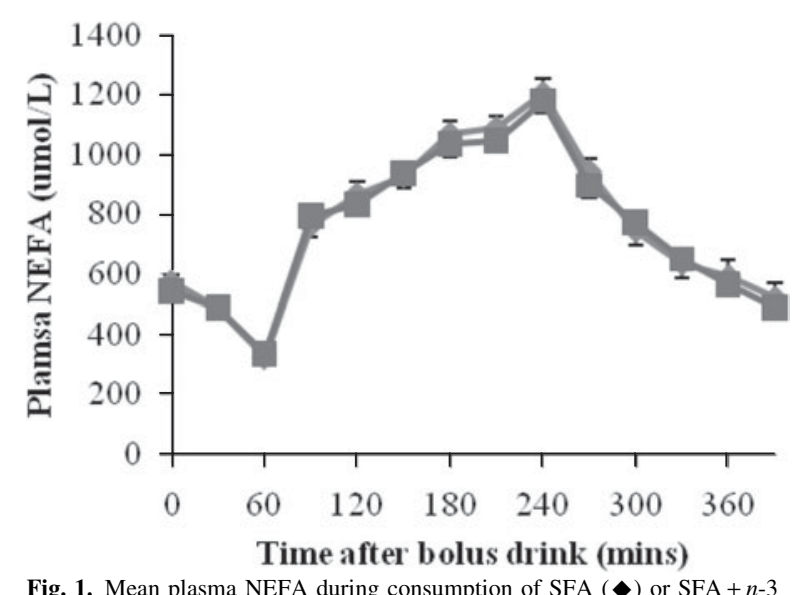

\begin{tabular}{lccc}
\hline & SFA & $n-3$ PUFA & $P$ value \\
\hline Males $(n$ 30) & & & \\
SI (mg/kg bw/min) & $7.2(0.4)$ & $7.8(0.4)$ & $0.05^{*}$ \\
SI (mg/kg ffm/min) & $8.5(0.4)$ & $9.3(0.4)$ & $0.04^{*}$ \\
Females $(n$ 30) & & & \\
SI (mg/kg bw/min) & $5.6(0.4)$ & $5.3(0.3)$ & 0.24 \\
SI (mg/kg ffm/min) & $7.9(0.6)$ & $7.6(0.5)$ & 0.28 \\
Total $(n$ 60) & & & \\
SI $(\mathrm{mg} / \mathrm{kg} \mathrm{bw} / \mathrm{min})$ & $6.4(0.3)$ & $6.6(0.3)$ & 0.35 \\
SI (mg/kg ffm/min) & $8.2(0.4)$ & $8.4(0.3)$ & \\
\hline
\end{tabular}

Mean (SEM). *Paired $t$ tests; statistically significant at $P \leq 0.05$.

Fig. 1. Mean plasma NEFA during consumption of SFA $(\bullet)$ or SFA $+n-3$

PUFA (ם), $n$ 48. Error bars represent SEM.

Plasma NEFA increased with the heparin infusion and subsequently declined during the HI clamp (Fig. 1); NEFA did not differ between the two study days. Insulin sensitivity was significantly greater during the consumption of $n$ - 3 PUFA than SFA for males but not females (Table).

Our data suggest that the substitution of a relatively small amount of SFA for $n$-3 PUFA during oral fat feeding with heparin infusion acutely increases SI in males. The reasons for the gender-specific effect are unclear, but there is some evidence to indicate that men may be more sensitive to the effects of lipid-induced impairments in $\mathrm{SI}^{(3,4)}$. Long-term trials are needed to investigate whether these acute study results could infer any benefit of dietary fish oil supplementation in improving SI in males.

The authors gratefully acknowledge funding from BBSRC, Unilever and FRST (New Zealand).

1. Belfort R et al. (2005) Diabetes 54, 1640-1648.

2. Beysen C et al. (2003) Am J Physiol Endocrinol Metab 284, E18-E24.

3. Frias JP et al. (2001) Diabetes 50, 1344-1350.

4. Masharani U, Goldfine ID \& Youngren JF (2009) Metabolism 58, 1602-1608. 